Research Article

\title{
Simultaneous Determination of Ropivacaine and 3-Hydroxy Ropivacaine in Cerebrospinal Fluid by UPLC-MS/MS
}

\author{
Siyuan Chen, ${ }^{1}$ Jianshe Ma, ${ }^{1,2}$ Xianqin Wang $\mathbb{D}^{1,2}$ and Quan Zhou $\mathbb{D}^{3}$ \\ ${ }^{1}$ Institute of Forensic Science, Wenzhou Medical University, Wenzhou 325000, China \\ ${ }^{2}$ Analytical and Testing Center, School of Pharmaceutical Sciences, Wenzhou Medical University, Wenzhou 325035, China \\ ${ }^{3}$ The Laboratory of Clinical Pharmacy, The People's Hospital of Lishui, Lishui 323000, China \\ Correspondence should be addressed to Xianqin Wang; lankywang@foxmail.com and Quan Zhou; 15057538800@163.com
}

Received 15 September 2020; Revised 19 November 2020; Accepted 25 November 2020; Published 4 December 2020

Academic Editor: Elena Baralla

Copyright (c) 2020 Siyuan Chen et al. This is an open access article distributed under the Creative Commons Attribution License, which permits unrestricted use, distribution, and reproduction in any medium, provided the original work is properly cited.

In this paper, a UPLC-MS/MS method was developed for the determination of ropivacaine and its metabolite 3-hydroxy ropivacaine in cerebrospinal fluid. The cerebrospinal fluid was processed by ethyl acetate liquid-liquid extraction. The multiple reaction monitoring $(\mathrm{MRM})$ mode was used for quantitative analysis by monitoring the transitions of $\mathrm{m} / \mathrm{z} 275.3 \rightarrow 126.2$ for ropivacaine, $\mathrm{m} / \mathrm{z} 291.0 \rightarrow 126.0$ for 3-hydroxy ropivacaine, and $\mathrm{m} / \mathrm{z} 290.2 \rightarrow 198.2$ for the internal standard. Standard curves for ropivacaine and 3-hydroxy ropivacaine in cerebrospinal fluid were conducted over the concentration range of $0.2-$ $2000 \mathrm{ng} / \mathrm{mL}$, demonstrating excellent linearity, and the lower limit of quantification was $0.2 \mathrm{ng} / \mathrm{mL}$. The intraday precision of ropivacaine and 3-hydroxy ropivacaine was less than $11 \%$, while the interday precision was less than $7 \%$. The accuracy ranged between $87 \%$ and $107 \%$, the average extraction efficiency was higher than $79 \%$, and the matrix effect was between $89 \%$ and $98 \%$. The developed method was then applied to a case of suspected poisoning of ropivacaine.

\section{Introduction}

Ropivacaine, which exists as the pure $S$-(-)-enantiomer, is a long-acting amide local anesthetic that can provide different effects at different doses [1]. At a concentration of $0.2 \%$, it exhibits a stronger sensory nerve block and almost no motor nerve block, whereas at a concentration of $0.75 \%$, it can the block motor nerve function $[2,3]$. The fat solubility of this product is greater than lidocaine but less than bupivacaine, and its anesthetic strength is 8 times that of procaine $[4,5]$. After epidural injection, the absorption is biphasic, and the fast and slow phase $t_{1 / 2}$ 's are $14 \mathrm{~min}$ and $4 \mathrm{~h}$, respectively. During bilateral intercostal nerve blocks, its absorption into the blood is faster than with epidural injection. Ropivacaine is mainly metabolized in the liver, and its metabolites have a weak local anesthetic effect [6]. Clinically, it is mainly used for regional block anesthesia and epidural anesthesia $[7,8]$. It is also used for regional block analgesia, such as analgesia after epidural surgery or labor [9]. Adverse reactions that can occur during epidural anesthesia are hypotension, bradycardia, nausea, and anxiety. When the blood concentration is too high, ropivacaine exhibits a dual effect by inhibiting and exciting the central nervous system, as well as inhibiting cardiac excitation, and prolonging the conduction of the cardiovascular system.

At present, various methods such as HPLC-UV [10], HPLC-DAD [11], LC-MS, or LC-MS/MS [12-20] have been developed for measuring the concentration of ropivacaine in biological fluids. The limits of quantitation of these methods ranged between $2.5 \mathrm{ng} / \mathrm{ml}$ and $75 \mathrm{ng} / \mathrm{mL}$. However, to the best of our knowledge, none of these studies reported the quantitation of ropivacaine and its metabolite 3-hydroxy ropivacaine in cerebrospinal fluid by UPLC-MS/MS. In this paper, an UPLC-MS/MS method was developed for the determination of ropivacaine and its metabolite 3-hydroxy ropivacaine in cerebrospinal fluid, which was then applied to a case of suspected poisoning of ropivacaine. 


\section{Materials and Methods}

2.1. Chemicals. Ropivacaine, 3-hydroxy ropivacaine, and diazepam-d5 (internal standard), all with purities $>98 \%$ (Figure 1), were purchased from Sigma-Aldrich Co. LLC (St. Louis, MO, USA). Acetonitrile and methanol (HPLC grade) were purchased from Merck (Darmstadt, Germany). Ultrapure water was prepared by the Millipore Milli-Q purification system (Bedford, MA, USA).

2.2. Instruments and Methods. The UPLC-MS/MS was composed of an ACQUITY H-Class UPLC and XEVO TQSmicro triple quadrupole mass spectrometer (Waters Corp, Milford, MA, USA). A UPLC BEH C18 column $(2.1 \mathrm{~mm} \times 50 \mathrm{~mm}, 1.7 \mu \mathrm{m}$, Waters, USA) was used as the stationary phase, and the column temperature was set at $40^{\circ} \mathrm{C}$. The mobile phase consisted acetonitrile and $10 \mathrm{mmol} / \mathrm{L}$ ammonium acetate (containing $0.1 \%$ formic acid) in water. The mobile phase was gradient eluted with a flow rate of $0.4 \mathrm{~mL} / \mathrm{min}$. From 0 to $0.2 \mathrm{~min}$, acetonitrile was $15 \%$ isocratic; from $0.2 \mathrm{~min}$ to $1.2 \mathrm{~min}$, acetonitrile was increased from $15 \%$ to $85 \%$; from $1.2 \mathrm{~min}$ to $2.1 \mathrm{~min}$, acetonitrile was $85 \%$ isocratic; from $2.1 \mathrm{~min}$ to $2.4 \mathrm{~min}$, acetonitrile was decreased from $85 \%$ to $15 \%$; from $2.4 \mathrm{~min}$ to $4.0 \mathrm{~min}$, acetonitrile was $15 \%$ isocratic.

The desolvation gas (nitrogen) was set to $850 \mathrm{~L} / \mathrm{h}$, and the cone gas was set to $50 \mathrm{~L} / \mathrm{h}$. The capillary voltage was set to $1.5 \mathrm{kV}$, and the desolvation temperature was $450^{\circ} \mathrm{C}$. The MRM mode was used for quantitative analysis. The selected MRM transitions were as follows: $\mathrm{m} / \mathrm{z} 275.3 \rightarrow 126.2$ (quantification) and $\mathrm{m} / \mathrm{z} 275.3 \rightarrow 84.3$ (confirmation) for ropivacaine (cone voltage $30 \mathrm{~V}$, collision voltage $20 \mathrm{~V}$ ); m/z 291.0 $\rightarrow 126.0$ (quantification) and $291.0 \rightarrow 98.3$ (confirmation) for 3-hydroxy ropivacaine (cone voltage $30 \mathrm{~V}$, collision voltage $20 \mathrm{~V}$ ); m/z $290.2 \rightarrow 198.2$ (quantification) and $290.2 \rightarrow$ 154.0 (confirmation) for diazepam-d5 (cone voltage $25 \mathrm{~V}$, collision voltage $30 \mathrm{~V}$ ) (Figure 2).

2.3. Preparation of Reference Solutions. Stock solutions of ropivacaine $(1.0 \mathrm{mg} / \mathrm{mL}), \quad 3$-hydroxy ropivacaine $(0.1 \mathrm{mg} / \mathrm{mL})$, and diazepam-d5 $(0.1 \mathrm{mg} / \mathrm{mL})$ were prepared in methanol. The stock solutions were diluted with methanol to prepare a series of standard working solutions, and all solutions were stored at $4^{\circ} \mathrm{C}$ until use.

2.4. Standard Curve Preparation. Blank cerebrospinal fluid was mixed with an appropriate amount of the standard working solutions to prepare a curve for ropivacaine and 3hydroxy ropivacaine with concentrations of $0.2,1,5,20$, $100,500,1000$, and $2000 \mathrm{ng} / \mathrm{mL}$ in cerebrospinal fluid. Quality control (QC) samples $(0.5,90$, and $1500 \mathrm{ng} / \mathrm{mL})$ were prepared in the same manner as the standard curve samples. The standard curve samples were treated by ethyl acetate liquidliquid extraction and analyzed by UPLC-MS/MS.

2.5. Sample Processing. Cerebrospinal fluid $(100 \mu \mathrm{L})$, the internal standard solution $(10 \mu \mathrm{L}$ of a $0.5 \mu \mathrm{g} / \mathrm{mL}$ solution), and ethyl acetate $(2 \mathrm{~mL})$ were added to a $5 \mathrm{~mL}$ centrifugation tube, which was then vortexed for $1.0 \mathrm{~min}$. After centrifugation $\left(3000 \mathrm{rpm}, 4^{\circ} \mathrm{C}, 10 \mathrm{~min}\right)$, the supernatant $(1.5 \mathrm{~mL})$ was removed and dried under a stream of air and then reconstituted in $100 \mu \mathrm{L}$ of methanol. The resulting solution $(80 \mu \mathrm{L})$ was transferred into the inner liner of the injection bottle, $2 \mu \mathrm{L}$ of which was injected into the UPLC-MS/MS for analysis.

2.6. Method Validation. The validation method was established in accordance with the US Food and Drug Administration Bioanalysis Method Validation Guidelines [21]. The selectivity of the method was evaluated by analyzing blank cerebrospinal fluid, and the blank cerebrospinal fluid spiked with ropivacaine, 3-hydroxy ropivacaine, and the internal standard by UPLC-MS/MS. For the standard curves, the standard solutions $(0.2,1,5,20,100,500,1000$, and $2000 \mathrm{ng} / \mathrm{mL}$ ) of ropivacaine and 3-hydroxy ropivacaine were prepared in cerebrospinal fluid. Under the same conditions, the area under each peak was measured, a standard curve was drawn with the peak area plotted against the concentration, and the linearity of the standard curve was evaluated. The lower limit of quantitation (LLOQ) was defined as the lowest concentration that could be detected with a signalto-noise ratio $(\mathrm{S} / \mathrm{N})$ exceeding 10 . The precision and accuracy of the LLOQ should be less than $20 \%$ and within $80 \%$ $120 \%$, respectively. The detection limit of the signal-tonoise ratio was 3 .

The precision and accuracy were evaluated by measuring QC samples at three separate concentrations in six replicates. Precision is expressed as a relative standard deviation (RSD), and the intraday and interday precisions were determined by measuring QC samples at three concentration levels on three consecutive days. The intraday and interday accuracies were reported based on the average value of the QC samples at the three different concentrations and the true value for the three consecutive days. The extraction efficiency was evaluated by comparing the measured peak area of the low-, medium-, and high-concentration QC samples to the corresponding standard peak area. The matrix effect was evaluated by comparing the peak area of the low-, medium-, and highconcentration standard solution in the blank cerebrospinal fluid after sample treatment to the peak area of the corresponding standard solution.

To confirm the ability to measure the diluted samples from beyond the upper limit of quantitation to within the calibration concentration range [22], six aliquots of ropivacaine $(20,000 \mathrm{ng} / \mathrm{mL})$ were diluted 20 -fold with blank cerebrospinal fluid for analysis. The peak area of the standard $(20,000 \mathrm{ng} / \mathrm{mL}$ in cerebrospinal fluid) after the 20 -fold dilution was compared to that of $1000 \mathrm{ng} / \mathrm{mL}$ undiluted sample. The acceptance criteria for ropivacaine consisted a precision (RSD) $\leq 15 \%$ and an accuracy of $85-115 \%$ after all six determinations [23].

The stability of ropivacaine and 3-hydroxy ropivacaine in cerebrospinal fluid was investigated by analyzing the QC samples of three low-, medium-, and high-concentration levels placed under three storage conditions, which include long-term stability $\left(-20^{\circ} \mathrm{C}, 30\right.$ days $)$, short-term stability ( $2 \mathrm{~h}$ at room temperature), and freeze-thaw stability ( 3 consecutive freezing and thawing cycles for 3 days $)\left(-20^{\circ} \mathrm{C}\right.$ to room temperature). 


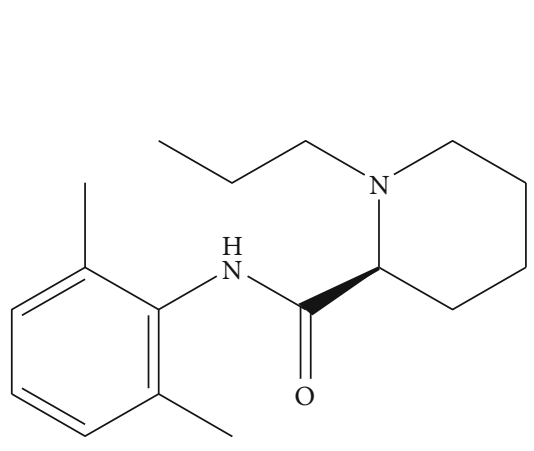

(a)

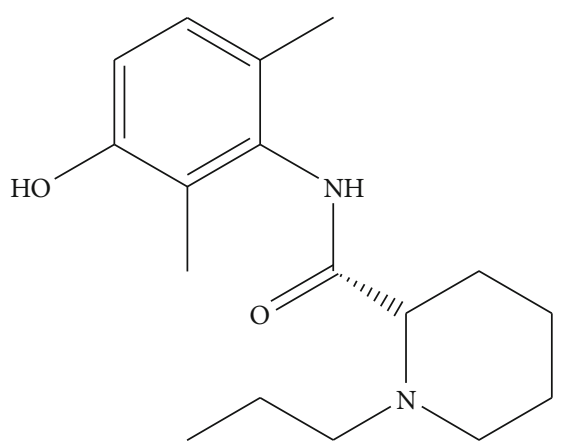

(b)<smiles>[2H]c1c([2H])c(P)c(P)c(C2=NCC(=O)N(C)c3ccc(Cl)cc32)c1[2H]</smiles>

(c)

FIgURE 1: Chemical structures of ropivacaine (a), 3-hydroxy ropivacaine (b), and diazepam-d5 (c).

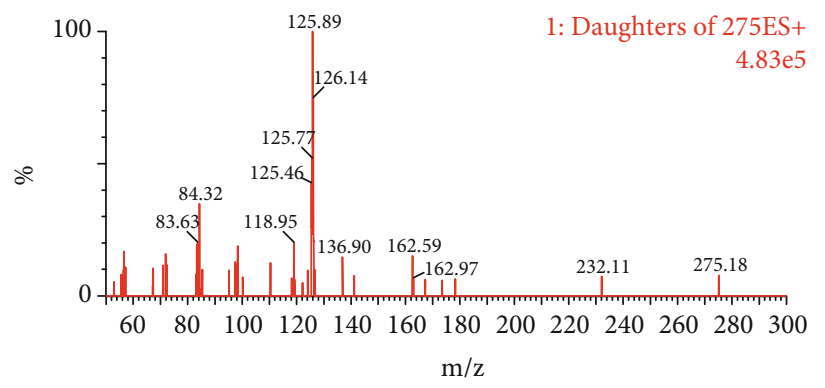

(a)

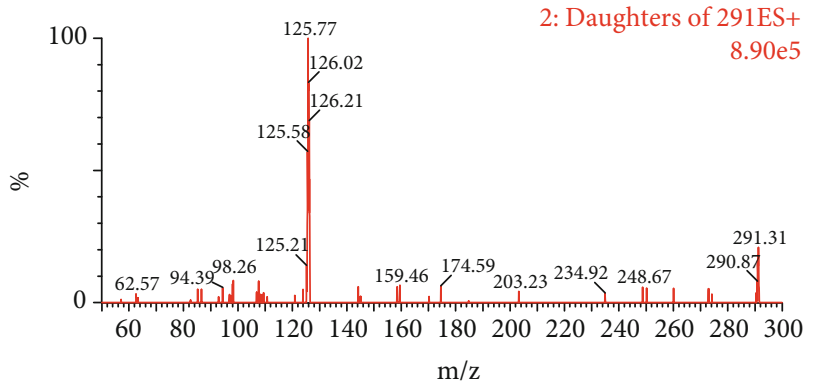

(b)

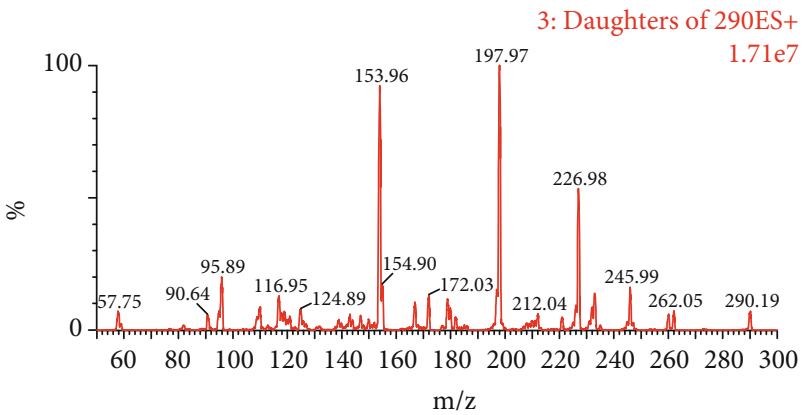

(c)

FIGURE 2: Mass spectra of ropivacaine (a), 3-hydroxy ropivacaine (b), and diazepam-d5 (c) in 10 mmol/L ammonium acetate (containing $0.1 \%$ formic acid) in water and acetonitrile $(1: 1, v / v)$.

\section{Result and Discussion}

3.1. Method Development. The choice of positive and negative mode for ESI is often evaluated in methodology [2426]. The experiment found that the ESI positive ion mode was more sensitive than the negative ion mode. After optimization of the mass spectrometry conditions, the appropriate cone voltage and collision voltage were selected as the mass spectrometry detection parameters, and the fragment peaks with high relative intensities were selected as the quantitative ion pairs.

The liquid chromatography conditions should be chosen in order to effectively separate endogenous interfering sub- stances [27-29] from ropivacaine, 3-hydroxy ropivacaine, and internal standard. During the method development, a series of mobile phases were examined and compared: methanol or acetonitrile (containing $0.1 \%$ formic acid), $10 \mathrm{mmol} / \mathrm{L}$ ammonium acetate buffer solution (containing $0.1 \%$ formic acid) in acetonitrile or methanol, and $10 \mathrm{mmol} / \mathrm{L}$ ammonium acetate buffer solution (containing $0.05 \%$ ammonia) in methanol or acetonitrile. Under comprehensive comparison, the gradient elution effect of $10 \mathrm{mmol} / \mathrm{L}$ ammonium acetate buffer solution (containing $0.1 \%$ formic acid) in acetonitrile afforded the best results, such that the peak shapes of ropivacaine and 3-hydroxy ropivacaine were narrow and the retention times were adjectives. 

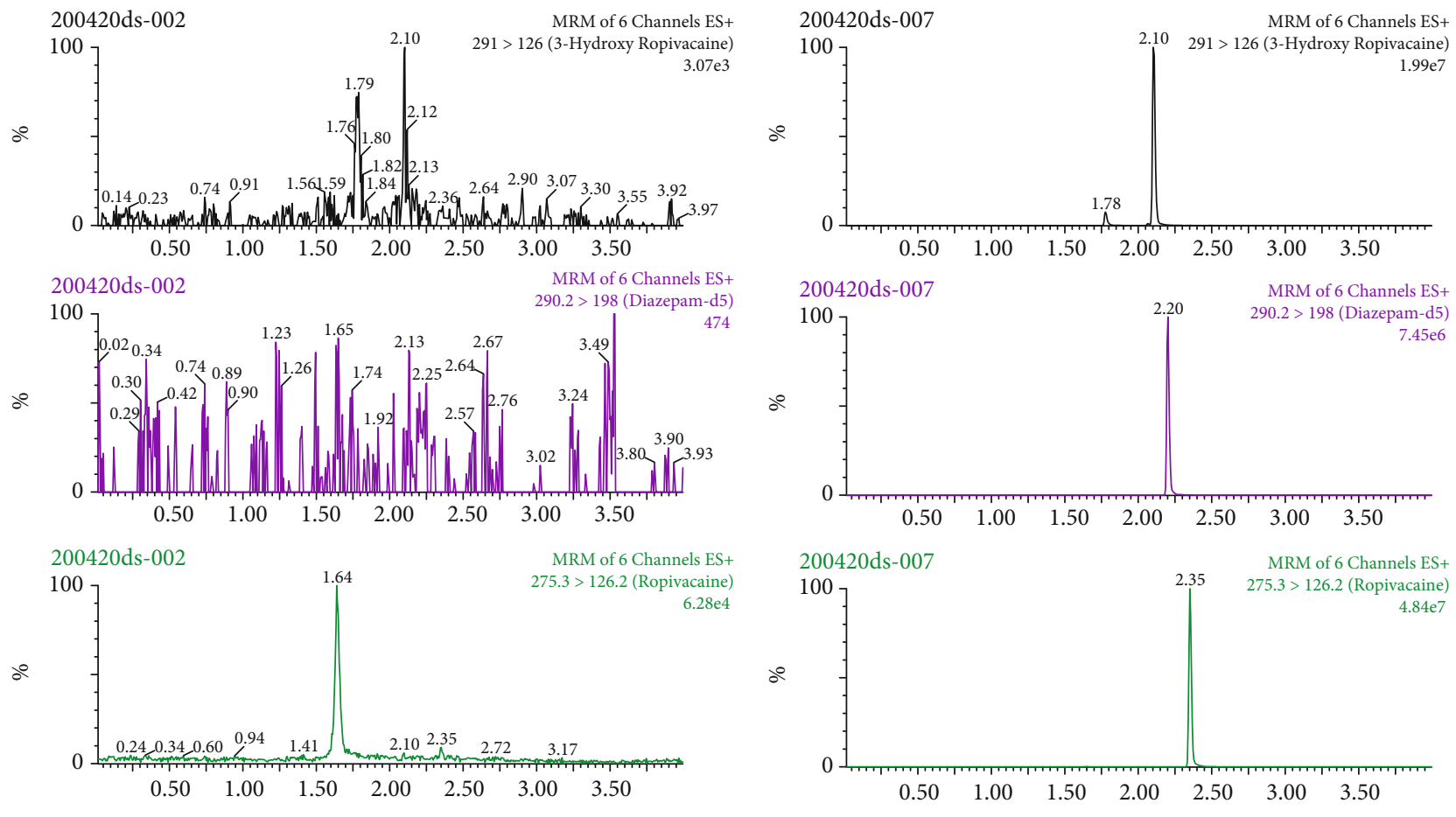

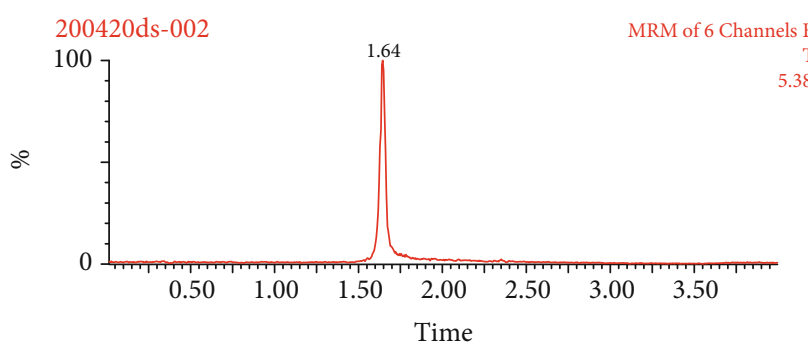

(a)

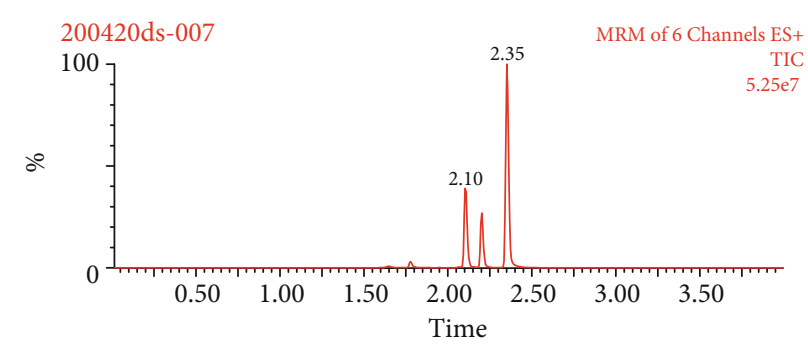

(b)

Figure 3: UPLC-MS/MS chromatograms of ropivacaine, 3-hydroxy ropivacaine, and diazepam-d5 in cerebrospinal fluid. (a) Blank cerebrospinal fluid. (b) Blank cerebrospinal fluid spiked with ropivacaine, 3-hydroxy ropivacaine, and diazepam-d5.

3.2. Method Validation. The typical UPLC-MS/MS chromatograms of blank cerebrospinal fluid, fortified cerebrospinal fluid with ropivacaine, 3-hydroxy ropivacaine, and the internal standard are shown in Figure 3. The retention times of ropivacaine, 3-hydroxy ropivacaine, and the internal standard were $2.35 \mathrm{~min}, 2.10 \mathrm{~min}$, and $2.20 \mathrm{~min}$, respectively. No obvious coextractives interfered with the detection.

The standard curves of ropivacaine and 3-hydroxy ropivacaine in cerebrospinal fluid were acquired over the concentration range of $0.2-2000 \mathrm{ng} / \mathrm{mL}$. The equations for the standard curve of ropivacaine and 3-hydroxy ropivacaine were $y=0.4069 x+0.2476, r=0.9998$, and $y=0.5768 x+$ $0.3461, r=0.9992$, where $y$ represents the ratio of the peak area of ropivacaine and 3-hydroxy ropivacaine to the internal standard and $x$ represents the concentration of ropivacaine and 3-hydroxy ropivacaine in cerebrospinal fluid. The LLOQ of ropivacaine and 3-hydroxy ropivacaine in cerebrospinal fluid was determined to be $0.2 \mathrm{ng} / \mathrm{mL}$. The detection limit of ropivacaine and 3-hydroxy ropivacaine in cerebrospinal fluid was $0.05 \mathrm{ng} / \mathrm{mL}$, and the signal-to-noise ratio was 3 .

It can be seen from Table 1 that the intraday precision of ropivacaine and 3-hydroxy ropivacaine was less than $11 \%$, the interday precision was less than $7 \%$, and the accuracy range was between $87 \%$ and $107 \%$. In addition, the average extraction efficiency was higher than $79 \%$, and the matrix effect was between $89 \%$ and $98 \%$. It was shown that the established UPLC-MS/MS method met the requirements for the analysis of biological samples of ropivacaine and 3-hydroxy ropivacaine with respect to precision, accuracy, extraction efficiency, and matrix effect.

After the 20 -fold dilution of the six aliquots of ropivacaine $(20,000 \mathrm{ng} / \mathrm{mL})$ in cerebrospinal fluid, the accuracy $(n=6)$ was identified as $98.5 \%$, and the precision (RSD, $n=$ 6) was $5.7 \%$. These results met the acceptance criteria, suggesting that samples exceeding the calibration curve concentrations can be diluted 20-fold to achieve concentrations within the range of the assay.

After analysis of the three stability conditions, which consisted of room temperature for 2 hours, $-20^{\circ} \mathrm{C}$ for 30 days, and 3 freezing and thawing cycles, the accuracy of ropivacaine and 3-hydroxy ropivacaine in cerebrospinal fluid was between $85 \%$ and $109 \%$, and the RSD was within $12 \%$. These results indicate that ropivacaine and 3-hydroxy ropivacaine were both stable. 
TABle 1: Precision, accuracy, extraction efficiency, and matrix effect data of ropivacaine and 3-hydroxy ropivacaine in cerebrospinal fluid $n=6$.

\begin{tabular}{|c|c|c|c|c|c|c|c|}
\hline \multirow{2}{*}{ Compound } & \multirow{2}{*}{ Concentration $(\mathrm{ng} / \mathrm{mL})$} & \multicolumn{2}{|c|}{ Accuracy (\%) } & \multicolumn{2}{|c|}{ Precision RSD (\%) } & \multirow{2}{*}{ Matrix effect } & \multirow{2}{*}{ Extraction efficiency } \\
\hline & & Intraday & Interday & Intraday & Interday & & \\
\hline \multirow{4}{*}{ Ropivacaine } & 0.2 & 91.9 & 93.5 & 10.0 & 6.6 & 97.1 & 80.2 \\
\hline & 0.5 & 93.2 & 99.2 & 6.8 & 5.9 & 94.9 & 84.1 \\
\hline & 90 & 106.2 & 106.6 & 2.7 & 3.4 & 96.3 & 87.9 \\
\hline & 1500 & 101.2 & 100.4 & 3.1 & 3.8 & 93.4 & 87.2 \\
\hline \multirow{4}{*}{ 3-Hydroxy ropivacaine } & 0.2 & 88.7 & 87.1 & 7.7 & 3.3 & 90.9 & 79.9 \\
\hline & 0.5 & 105.6 & 96.1 & 4.3 & 5.5 & 93.9 & 83.3 \\
\hline & 90 & 95.9 & 103.4 & 5.3 & 1.8 & 92.5 & 85.0 \\
\hline & 1500 & 102.2 & 97.1 & 4.3 & 5.5 & 89.8 & 84.3 \\
\hline
\end{tabular}

3.3. Application. A woman with pain in the right elbow was diagnosed with tennis elbow and cervical spondylosis after a physical examination, cervical X-ray, and cervical magnetic resonance imaging. The patient was then given local injections, which consisted $20 \mathrm{mg}$ of ropivacaine mesylate, $20 \mathrm{mg}$ of sodium hyaluronate, $3.0 \mathrm{~g}$ of adenosine cobaltamine, and $10 \mathrm{mg}$ of tramalin dissolved in $10 \mathrm{~mL}$ of saline, in the facet joints of the cervical spine to improve elbow pain. During injection therapy, the patient experienced chest tightness and difficulty breathing. The treating physician immediately stopped the injection and gave oxygen inhalation, mouthto-mouth artificial respiration, injection of respiratory stimulants, and continuous-breathing mask pressure to maintain respiration. After extracorporeal cardiac compressions, an injection of $1 \mathrm{mg}$ norepinephrine, continuous cardiac compression, balloon breathing, and other treatments, rescue continued for about 40 minutes. At last, the patient's breathing and heartbeat showed no signs of recovery, and the rescue was declared invalid. The disputes over the cause of death requested ropivacaine testing of the cerebrospinal fluid. The concentrations of ropivacaine and 3-hydroxy ropivacaine in the cerebrospinal fluid were $22.8 \mu \mathrm{g} / \mathrm{mL}$ and $0.57 \mathrm{ng} / \mathrm{mL}$ by the developed UPLC-MS/MS, respectively. According to the literature, a concentration of $2.2 \mu \mathrm{g} / \mathrm{mL}$ of ropivacaine in plasma was reported to be toxic [30], whereas the toxic concentration in cerebrospinal fluid was unknown.

\section{Conclusion}

In this study, a sensitive, rapid, and selective UPLC-MS/MS method was established for the determination of ropivacaine and 3-hydroxy ropivacaine in cerebrospinal fluid with a linear range of $0.2-2000 \mathrm{ng} / \mathrm{mL}$. UPLC-MS/MS has a faster analysis time and higher sensitivity than traditional HPLC. It only takes $4 \mathrm{~min}$ to complete the analysis of one cerebrospinal fluid sample, which could save a significant amount of time and resources. It was also successfully applied to a ropivacaine poisoning case.

\section{Data Availability}

The data used to support the findings of this study are included within the article.

\section{Conflicts of Interest}

The authors declare that there is no conflict of interest regarding the publication of this paper.

\section{Acknowledgments}

This work was supported by the Zhejiang Provincial Natural Science Foundation of China (LY19H230001).

\section{References}

[1] G. Kuthiala and G. Chaudhary, "Ropivacaine: a review of its pharmacology and clinical use," Indian Journal of Anaesthesia, vol. 55, no. 2, pp. 104-110, 2011.

[2] D. Simpson, M. P. Curran, V. Oldfield, and G. M. Keating, "Ropivacaine: a review of its use in regional anaesthesia and acute pain management," Drugs, vol. 65, no. 18, pp. 26752717, 2005.

[3] M. Wille, "Intrathecal use of ropivacaine: a review," Acta Anaesthesiologica Belgica, vol. 55, no. 3, pp. 251-259, 2004.

[4] Y. Beilin and S. Halpern, "Focused review: ropivacaine versus bupivacaine for epidural labor analgesia," Anesthesia and Analgesia, vol. 111, no. 2, pp. 482-487, 2010.

[5] Y. Kaufman, I. Hirsch, L. Ostrovsky et al., "Pain relief by continuous intraperitoneal nebulization of ropivacaine during gynecologic laparoscopic surgery-a randomized study and review of the literature," Journal of Minimally Invasive Gynecology, vol. 15, no. 5, pp. 554-558, 2008.

[6] T. G. Hansen, "Ropivacaine: a pharmacological review," Expert Review of Neurotherapeutics, vol. 4, no. 5, pp. 781-791, 2014.

[7] Y. Zhang, L. Liu, L. Zheng et al., "Comparison of effectiveness of ropivacaine infusion regimens for continuous femoral nerve block for recovery after total knee arthroplasty: a randomized double-blind trial," Journal of Pain Research, vol. 13, pp. 997-1005, 2020.

[8] L. Huang, L. Zheng, B. Wu et al., "Effects of ropivacaine concentration on analgesia after ultrasound-guided serratus anterior plane block: a randomized double-blind trial," Journal of Pain Research, vol. 13, pp. 57-64, 2020.

[9] Y. Wang and $\mathrm{M} . \mathrm{Xu}$, "Comparison of ropivacaine combined with sufentanil for epidural anesthesia and spinal-epidural anesthesia in labor analgesia," BMC Anesthesiology, vol. 20, no. 1, p. 1, 2020. 
[10] N. Rifai, O. Hsin, T. Hope, and M. Sakamoto, "Simultaneous measurement of plasma ropivacaine and bupivacaine concentrations by HPLC with UV detection," Therapeutic Drug Monitoring, vol. 23, no. 2, pp. 182-186, 2001.

[11] A. Salmeron-Garcia, N. Navas, A. Martin, E. Roman, J. Cabeza, and L. F. Capitan-Vallvey, "Determination of tramadol, metamizole, ropivacaine, and bupivacaine in analgesic mixture samples by HPLC with DAD detection," Journal of Chromatographic Science, vol. 47, no. 3, pp. 231-237, 2009.

[12] E. Lamy, F. Fall, L. Boigne, K. Gromov, N. Fabresse, and S. Grassin-Delyle, "Validation according to European and American regulatory agencies guidelines of an LC-MS/MS method for the quantification of free and total ropivacaine in human plasma," Clinical Chemistry and Laboratory Medicine, vol. 58, no. 5, pp. 701-708, 2020.

[13] M. Abdel-Rehim, M. Dahlgren, and L. Blomberg, "Quantification of ropivacaine and its major metabolites in human urine samples utilizing microextraction in a packed syringe automated with liquid chromatography-tandem mass spectrometry (MEPS-LC-MS/MS)," Journal of Separation Science, vol. 29, no. 11, pp. 1658-1661, 2006.

[14] Z. Cobb and L. I. Andersson, "Determination of ropivacaine in human plasma using highly selective molecular imprint-based solid phase extraction and fast LC-MS analysis," Analytical and Bioanalytical Chemistry, vol. 383, no. 4, pp. 645-650, 2005.

[15] T. Breindahl, O. Simonsen, and K. Andreasen, "Columnswitching HPLC-MS/MS analysis of ropivacaine in serum, ultrafiltrate and drainage blood for validating the safety of blood reinfusion," Journal of Chromatography B, vol. 878, no. 1, pp. 76-82, 2010.

[16] K. Sawaki, M. Okubo, T. Shimomiya et al., "Evaluation of high-performance liquid chromatography and mass spectrometry method for pharmacokinetic study of local anesthetic ropivacaine in plasma," Biomedical Research, vol. 30, no. 6, pp. 319-324, 2009.

[17] O. Mathieu, D. Hillaire-Buys, C. Dadure, F. Barnay, J. C. Mathieu-Daude, and F. Bressolle, "Liquid chromatographyelectrospray mass spectrometry determination of free and total concentrations of ropivacaine in human plasma," Journal of Chromatography B, vol. 831, no. 1-2, pp. 91-98, 2006.

[18] M. Abdel-Rehim, Z. Altun, and L. Blomberg, "Microextraction in packed syringe (MEPS) for liquid and gas chromatographic applications. Part II-determination of ropivacaine and its metabolites in human plasma samples using MEPS with liquid chromatography/tandem mass spectrometry," Journal of Mass Spectrometry, vol. 39, no. 12, pp. 1488-1493, 2004.

[19] S. K. Bergstrom and K. E. Markides, "On-line coupling of microdialysis to packed capillary column liquid chromatography-tandem mass spectrometry demonstrated by measurement of free concentrations of ropivacaine and metabolite from spiked plasma samples," Journal of Chromatography B, vol. 775, no. 1, pp. 79-87, 2002.

[20] M. Stumpe, N. S. Morton, and D. G. Watson, "Determination of free concentrations of ropivacaine and bupivacaine in plasma from neonates using small-scale equilibrium-dialysis followed by liquid chromatography-mass spectrometry," Journal of Chromatography B, vol. 748, no. 2, pp. 321-330, 2000.

[21] FDA, Guidance for Industry Analytical Procedures and Methods Validation for Drugs and Biologics2014, https://www.fdanews .com/ext/resources/files/02/02-19-14-Guidance.pdf.

[22] W. J. Ye, R. J. Chen, W. Sun et al., "Determination and pharmacokinetics of engeletin in rat plasma by ultra-high perfor- mance liquid chromatography with tandem mass spectrometry," Journal of Chromatography B, vol. 1060, pp. 144-149, 2017.

[23] A. Szeitz, J. Manji, K. W. Riggs, A. Thamboo, and A. R. Javer, "Validated assay for the simultaneous determination of cortisol and budesonide in human plasma using ultra high performance liquid chromatography-tandem mass spectrometry," Journal of Pharmaceutical and Biomedical Analysis, vol. 90, pp. 198-206, 2014.

[24] Q. Zhou, Z. G. Zhang, P. Geng, B. G. Huang, X. Q. Wang, and X. M. Yu, "Pharmacokinetics of ligustroflavone in rats and tissue distribution in mice by UPLC-MS/MS," Acta Chromatographica, vol. 32, no. 2, pp. 102-106, 2020.

[25] H. L. Xie, X. J. Lu, W. Q. Jin et al., "Pharmacokinetics of picroside I, II, III, IV in rat plasma by UPLC-MS/MS," Current Pharmaceutical Analysis, vol. 16, no. 4, pp. 438-445, 2020.

[26] G. T. Lin, Y. Y. Chen, Y. Yu, H. Z. Wang, X. Q. Wang, and L. M. Chen, "Pharmacokinetics of talatisamine in rat plasma by UPLC-MS/MS," Latin American Journal of Pharmacy, vol. 39, no. 6, pp. 1105-1109, 2020.

[27] L. Hu, L. Wang, J. Zhang et al., "Tissue distribution study of MGCD0103 in rat by UPLC-MS/MS," Latin American Journal of Pharmacy, vol. 35, no. 10, pp. 2288-2291, 2016.

[28] Y. Chen, Y. He, S. Yang et al., "Development and validation of a LC-MS assay for quantification of CI-994 (N-acetyldinaline) in rat plasma and its application to a pharmacolcinetic study," Latin American Journal of Pharmacy, vol. 35, no. 3, pp. 578$585,2016$.

[29] H. Wu, M. Lu, J. He et al., "Determination and pharmacokinetics and bioavailability of O-demethyl nuciferine in mice by UPLC-MS/MS," Acta Chromatographica, vol. 31, no. 3, pp. 222-227, 2019.

[30] R. J. Miller, A. J. Cameron, J. Dimech, R. J. Orec, and N. J. Lightfoot, "Plasma ropivacaine concentrations following local infiltration analgesia in total knee arthroplasty: a pharmacokinetic study to determine safety following fixed-dose administration," Regional Anesthesia and Pain Medicine, vol. 43, no. 4, pp. 347-351, 2018. 